\title{
LOS SOBRECOSTOS EN LOS EQUIPOS DE MEDICIÓN DEL PROCESO DE PINTURA DE LA EMPRESA RENAULT SOFASA, APLICANDO LA METODOLOGÍA KAIZEN
}

\author{
Result research on surgeons in the measurement equipment of the paint process of the Renault
} Sofasa company, applying the Kaizen methodology

\author{
Diego Alejandro Henao-Cárdenas \\ Ingeniero en Producción. Renexter Outsourcing de Renault -Sofasa. Medellín-Colombia. \\ diego.henao-renexter@renault.com \\ Germán Alberto Cano-Ortiz \\ Tecnólogo en Calidad. Instituto Tecnológico Metropolitano. Medellín-Colombia. \\ germancano@itm.edu.co \\ Jairo Alonso Palacio-Morales \\ Especialista en Educación Superior. Instituto Tecnológico Metropolitano. Medellín-Colombia. \\ jairopalacio@itm.edu.co
}

\begin{abstract}
Cómo citar / How to cite
Henao-Cárdenas, D.A., Cano-Ortiz, G.A. y Palacio-Morales, J.A. (2017). Los sobrecostos en los equipos de medición del proceso de pintura de la empresa Renault Sofasa, aplicando la metodología Kaizen. Revista CEA, 3(6), 77-92.
\end{abstract}

Recibido: 20 de abril de 2017

Aceptado: 30 de mayo de 2017

\section{Resumen}

En este artículo se presentan los resultados de la investigación sobre los factores que inciden en el retiro de los equipos de medición del proceso de pintura, de la empresa colombiana ensambladora de vehículos Renault Sofasa. Empleando métodos como el Kaizen y el Sistema de Aseguramiento Metrológico (SAM), se realiza un diagnóstico para determinar la pérdida económica generada por los sobrecostos ocasionados por los equipos de medición en etapas críticas del proceso productivo. Los resultados obtenidos por medio de esta investigación servirán de base para que la compañía desarrolle soluciones pertinentes, utilizando esta información como una herramienta para mitigar los sobrecostos generados en el departamento de producción. Los resultados obtenidos sugieren la importancia de contar con un método adecuado para controlar y mejorar los procesos, no solo en la industria automotriz, sino en todos los sectores económicos que utilicen equipos de medición y que estos se encuentren vinculados con las variables críticas de control y la elaboración de los productos.

Palabras clave: costos, sistema metrológico, mejoramiento continuo, Kaizen.

\begin{abstract}
This article presents the results of the investigation into the factors that affect the removal of the measuring equipment from the painting process of the Colombian company assembling Renault Sofasa vehicles. Using methods such as the Kaizen and the metrological assurance system (SAM), a diagnosis is made to determine the economic loss generated by the overcharges caused by measuring equipment at critical stages of the production process. The results obtained
\end{abstract}


through this research will serve as a basis for the company to develop relevant solutions, using this information as a tool to mitigate the cost overruns generated in the production department.

The results obtained suggest the importance of having an adequate method to control and improve the processes, not only in the automotive industry but also in all economic sectors that use measuring equipment and that these are linked to the critical variables of control and the elaboration of the products.

Keywords: costs, metrological system, continuous improvement Kaizen.

\section{INTRODUCCIÓN}

Todo producto tiene implícito un costo de elaboración. Cuando estos costos aumentan disminuye proporcionalmente el beneficio para las empresas y afecta al consumidor. Es por esto que las compañías buscan soluciones rápidas y eficientes para mitigar este impacto.

Históricamente la industria automotriz se caracteriza por ser pionera en el desarrollo y la utilización de diferentes metodologías y herramientas de mejoramiento, que permiten controlar y mejorar todos los procesos (Unger, 2004). De esta forma pueden mitigar los sobrecostos generados y atacar los problemas presentados durante la transformación de los productos. Una de las metodologías más populares es el Kaizen (mejoramiento continuo), que según Maarof \& Mahmud (2016) es un método para mejorar la competitividad en las empresas, por medio de una serie de aplicaciones. Esta herramienta japonesa es cada vez más implementada por las empresas que buscan ser más competitivas y reconocidas por sus altos estándares de calidad en el mercado global.
La industria automotriz innova constantemente sus sistemas de gestión de la calidad, lo que ha permitido mantener $y$ aumentar su productividad y rentabilidad, a pesar de los constantes retos de los mercados. Según la Organización Internacional de Constructores de Automóviles, en el año 2015 fueron producidos 90,780,583 vehículos a nivel mundial. Según la Asociación Nacional de Empresarios de Colombia (ANDI \& FENALCO, 2015), en Colombia operan ocho empresas ensambladoras, sin embargo, cuatro de ellas concentran el $96 \%$ de producción en términos de unidades, de la siguiente manera: General Motors Colmotores (marcas Isuzu, Volvo y Chevrolet), Sociedad de Fabricación de Automotores - SOFASA (marca Renault), Hino Motors Manufacturing S.A. (marca Hino grupo Toyota) y Fotón.

La Sociedad de Fabricación de Automotores S.A - SOFASA (marca Renault), empresa colombiana fundada en 1969 por el gobierno de Francia, con el objetivo de ensamblar vehículos de la marca Renault y estimular el desarrollo de una industria automotriz y autopartista en el país, ocupó los primeros lugares en producción con el $21 \%$ de participación del mercado a nivel nacional en el año 2015. A mayo de 2017 la marca Renault ocupa el segundo lugar en vehículos nuevos matriculados a nivel nacional (FENALCO, 2017), además este informe resalta que las ensambladoras que se encuentran en el país son las principales proveedoras del mercado interno automotriz, lo que demuestra la importancia de esta empresa en la economía a nivel nacional.

Una de las características principales de esta industria es su automatizada infraestructura, la cual requiere implementar métodos de control adaptativos a sus necesidades, permitiendo establecer parámetros de mejoramiento y control. El Sistema Aseguramiento Metrológico (SAM) permite a este tipo de organizaciones 
controlar todo el sistema medición y a su vez proporciona herramientas para evaluar los sobrecostos y los efectos que estos generan sobre la productividad de los procesos.

Los sobrecostos son definidos como la cantidad de dinero que se invierte y que excede lo presupuestado. Para el caso de esta investigación, la pérdida de dinero en los equipos de medición que intervienen en los diferentes procesos y que por diversos factores son dados de baja. En Restrepo (2016), se propone determinar los costos totales durante la vida útil de los equipo en el proceso, para con los resultados obtenidos tomar acciones correctivas que garanticen el mejoramiento continuo de los procesos y la rentabilidad de la compañía (Jimenez, 2007).

En este artículo se presentan los resultados de la investigación que tiene como objetivo diagnosticar los factores de incidencia que generan sobrecostos en los equipos de medición, basados en el sistema de aseguramiento metrológico de la empresa Renault-Sofasa en un periodo de 5 años, partiendo desde el costo de adquisición hasta los costos por pérdida y mal funcionamiento de los equipos. Los resultados de esta investigación permiten identificar los sobrecostos de los equipos de medición dados de baja en la empresa Renault-Sofasa en el proceso de pintura, con el propósito de determinar los factores de incidencia y calcular los costos de pérdida en los equipos retirados del proceso.

\section{MARCO DE REFERENCIA}

\section{Sistema de aseguramiento metrológico}

Con el fin de garantizar los buenos procedimientos de medición relacionados con los procesos y las competencias técnicas del personal ligado a la interpretación de las diversas magnitudes en la industria, se hace indispensable el contar con la correcta gestión de las operaciones de medición y la respectiva confirmación metrológica de los instrumentos empleados para determinar el cumplimiento de los requerimientos del aseguramiento Metrológico (Restrepo, 2016).

Con el propósito de aportar a la calidad de los resultados obtenidos en un sistema de mediciones, se da la aparición de estrategias que permiten realizar una adecuada gestión de las mediciones, asegurando que los equipos y los procesos de medición son adecuados para alcanzar la calidad del producto, minimizando el riesgo de obtener resultados de medición incorrectos. Una herramienta para la implementación de un sistema de aseguramiento metrológico es la Norma Técnica Colombiana 10012 (ICONTEC, 2003), que entregan un conjunto de elementos interrelacionados para la confirmación metrológica y el control de los diversos procesos de medición en la industria. En casos particulares como los laboratorios de inspección y ensayo, normas como la NTC ISO/IEC 17025 (ICONTEC, 2002) exigen el cumplimiento de la exactitud requerida y las especificaciones por parte de los equipos utilizados para los ensayos. Adicionalmente otras normativas apoyan los procesos de calidad en las organizaciones (ISO, 2007, 2008, 2015).

Un buen sistema de aseguramiento metrológico requiere de lo que se denomina como Operaciones de Confirmación Metrológica (OCM). Dichas operaciones permiten garantizar el cumplimiento de los requisitos y especificaciones técnicas por parte de los instrumentos de medición. La confirmación metrológica se desarrolla por medio de actividades como: mantenimiento, ajuste, verificación y calibración. Activadas en las cuales son de importancia la capacidad de medición y calibración y los errores permitidos. 
Una de las herramientas propias para la implementación de un sistema de aseguramiento metrológico es el ciclo de planificación, ejecución, verificación, acción PHVA. Cada una de las etapas del ciclo aporta elementos que pueden ser empleados para la optimización de diversos procesos productivos.

La planificación permite la definición de planes y procedimientos de confirmación metrológica para el cumplimento de los requisitos metrológicos del proceso de medición. En la etapa de ejecución se realiza el seguimiento de los parámetros definidos por el proceso de confirmación metrológica para determinar una adecuada supervisión de los resultados. Al igual que la planificación la etapa de verificación es importante dado que permite obtener la información del estado de los instrumentos de medición que aporta posteriormente a la confirmación en el sistema de aseguramiento metrológico.

Los sistemas de aseguramiento metrológico se han convertido en una herramienta transversal de gran importancia en diversos sectores económicos para la optimización de sus procesos en pro de la calidad y la disminución de sobrecostos, un ejemplo de ello son aplicaciones en la producción y uso de radiofármaco (Hernández, Oropesa, \& Serra, 2012). En el campo de las mediciones en equipos médicos (Rodríguez, 2013) y en las pruebas de ensayo no destructivos (López, Amezola, \& Elías, 2007) entre otros.

\section{Mejoramiento continuo KAIZEN}

Es considerada como una herramienta para la práctica del mejoramiento continuo. De origen oriental su aparición en occidente es proporcionado por Masaaki Imai, el cual plantea la generación de un método que es reconocido a nivel mundial como el principio fundamental de las estrategias competitivas a mediano y a largo plazo. El significado del Kaizen se deriva en dos palabras; KAl «cambio» y ZEN «bueno».

El Kaizen como cualquier tipo de filosofía de mejoramiento se basa en los siguientes principios:

- Los buenos procesos originan buenos resultados.

- Hablar con datos, gestionar con hechos

- Trabajo en equipo.

- Observación de los procesos para su posterior análisis y comprensión.

- Toma de medidas y correcciones luego de detectar la causa y raíz del problema.

- Lo que se mide, se puede controlar y mejorar.

Uno de los requerimientos más importantes se enfoca en la satisfacción plena del consumidor de un producto o servicio. Por tal razón, se requiere de métodos que potencialicen la disciplina y el respeto por el trabajo bien desarrollado. El método Kaizen se basa en filosofías que apoyadas en herramientas administrativas que ha sido implementada en diversas industrias occidentales en materia automotriz, relojería, cámaras fotográficas entre otras aplicaciones (Chirinos, Rivero, \& Méndez, 2015; Imai, 2003).

Esta filosofía tiene como objetivo principal mejorar la calidad en los procesos y reducir los costos de producción de las compañías utilizando como herramienta principal el circuito Deming o conocido como el ciclo PHVA (Planear, Hacer, Verificar y Actuar). Las etapas de este ciclo son definidas a continuación:

- Planear: es la primera etapa en la cual el equipo responsable planifica el objetivo, analiza el problema y define como se va a desarrollar la intervención de la no conformidad. 
- Hacer: en esta etapa se ejecutan las acciones tomadas y se lleva un registro de cada paso de las acciones desarrolladas.

- Verificar: secuencialmente se puede definir como la tercera etapa en la cual se realiza la verificación de los resultados obtenidos.

- Actuar: teniendo como base los resultados obtenidos, se realiza la toma de decisiones pertinentes de ser necesario para obtener algún cambio o mejora.

El ciclo PHVA es repetitivo, presentando óptimos resultados cuando todos los niveles de la organización presentan alto compromiso y disciplina. Dentro de la herramienta Kaizen se cuenta con una clasificación de técnicas de la misma filosofía, donde aparecen como destacadas las 5S, mantenimiento productivo total (TPM) y justo a tiempo (para Conesa, 2007).

Técnica 5 S: esta técnica de las Cinco Eses se deriva de las palabras Seiri, Seiton, Seiso, Seiketsu, y Shitsuke esta combinación traduce en pocas palabras eliminar lo innecesario.

- Seiri (clasificar): eliminar del entorno todos los elementos innecesarios para ejecutar una labor.

- Seiton (ordenar): establece la forma en que los materiales deben ser ubicados de tal manera que sea fácil de identificar.

- Seiso (limpieza): mantener el entorno limpio y eliminar los riegos laborales.

- Seiketsu (estandarizar): creación de un modo consistente y de realizar tareas 0 procedimientos

- Shitsuke (disciplina): convertir en hábito el empleo de los métodos establecidos.

Su filosofía se basa en el principio de contar con un lugar de trabajo limpio y seguro; este método pretende reducir pérdidas de tiempo por búsquedas, reducción de gastos por deterioro, eliminar fuentes de suciedad, mejorar la utilización de los espacios disponibles, entre otros.

\section{Técnica Mantenimiento Productivo total (TPM)}

Esta filosofía japonesa se enfoca en la eliminación de pérdidas asociadas con paros y lo relacionado a la calidad en los procesos. Esta herramienta permite asegurar la disponibilidad y confiabilidad prevista de las operaciones, de los equipos, y del sistema, mediante la aplicación de los conceptos de prevención, cero defectos, cero accidentes y participación total de las personas.

El TPM se fundamenta sobre seis pilares:

- Mejoras enfocadas.

- Mantenimiento autónomo.

- Mantenimiento planificado.

- Mantenimiento de calidad.

- Educación y entrenamiento.

- Seguridad y medio ambiente.

Dentro de las actividades del TPM se tienen:

- Diseño de actividades de mejora para aumentar la eficiencia de los equipos.

- Disposición de un sistema de mantenimiento autónomo.

- Establecimiento de necesidades de formación periódica para los empleados.

- Aprovechamiento del capital humano.

- Reducción de costos operativos.

- Seguimiento y control de indicadores.

\section{Justo a tiempo (Just-in-Time)}

Este sistema de producción comenzó su implementación en la línea de producción de Toyota. Esta herramienta define la forma de optimizar un sistema de producción, de tal modo que se entreguen las materias primas a la línea de producción en el momento indicado a medida que sea necesario. 
Los cuatro objetivos principales de esta técnica de trabajo son:

- Buscar la simplicidad.

- Diseñar sistemas para identificar problemas.

- Eliminar despilfarros.

- Poner en evidencia los problemas fundamentales.

Justo a tiempo implica producir solo lo necesario para cumplir las metas pedidas por el cliente. Adicionalmente, se busca producir el mínimo número de unidades en las menores cantidades posibles y en el último momento posible. El objetivo es eliminar la necesidad de almacenaje permitiendo que las existencias mínimas y suficientes se suministren justo a tiempo para reponer las que acaban de utilizarse y la eliminación del inventario de producto terminado.

Para las empresas del sector automotriz esta filosofía es muy indispensable, teniendo en cuenta el espacio utilizado al momento de realizar la transformación, esto garantiza que se minimicen los reprocesos generados por daños de la materia prima.

En la actualidad, el requerimiento es el mejoramiento de los estándares en la búsqueda de la competitividad de las empresas. Aquellas industrias que adopten este método de mejoramiento en todos los niveles de la estructura organizacional, contará con una nueva visión hacia el cambio afrontando cada día el reto de mejorar sus estándares de productividad. Renault Sofasa lleva ejecutando estas herramientas mencionadas desde hace aproximadamente 25 años. Todos sus procesos están estandarizados y continuamente buscan mejorar cada día. El compromiso del personal de la compañía es reconocido y consideran que siempre hay una mejor manera de hacer las cosas. Para esta investigación, se realiza el diagnóstico de factores de incidencia en los últimos 5 años aplicando el principio del Pareto. Dicho principio parte de realizar la división de una muestra en dos grupos de proporciones de 80 - 20. Al ser un principio basado en lo económico, se establece que el $20 \%$ de lo que entra o se invierte es generador del $80 \%$ de los resultados obtenidos. Lo anterior es equivalente a determinar que el $20 \%$ de las causas generan el $80 \%$ de las consecuencias (Kume, 1992).

\section{Costos del sistema de aseguramiento metrológico}

Una parte relevante de un sistema de aseguramiento metrológico es identificar los costos del sistema de mediciones, el cual no solo tiene en cuenta el costo de adquisición del equipo o instrumento de medida, si no todas aquellas acciones a las que son sometidas los equipos para garantizar la calidad en las mediciones, las cuales acarrean consigo un costo que en algunas ocasiones no se tenía contemplado al momento de adquirir los instrumentos de medición. Los siguientes costos son propuestos por (Restrepo, 2016) con el objetivo de determinar los costos totales durante el periodo de vida de los equipos.

\section{Costo de adquisición}

Costo inicial que se paga por bien o servicio, incluye las siguientes etapas de la compra:

- Evaluación del proveedor: costo de análisis de los proveedores y los equipos, teniendo en cuenta que se debe tener una persona destinada a ejecutar esta operación.

- Precio del producto: costo real del producto terminado.

- Transporte: costos por envío del producto, generalmente este costo se carga al consumidor. 
- Tiempo de despacho: costos generados por retrasos en la entrega del instrumento, por motivos que no tienen que ver con el proceso, etc.

\section{Costo de operación}

Estos costos son complejos de definir teniendo en cuenta que todos los instrumentos tienen gran variedad de elementos que comprometen la operatividad.

- Costos de documentación: estimar todos los costos generados que acarrean la gestión documental, como por ejemplo desarrollar informes de calibración, procedimientos de medición, informes de gestión.

\section{Costo de calibración}

Para garantizar una buena medición es necesario contar con los equipos e infraestructura adecuados, y que las mediciones sean trazables.

- Costo para la adquisición de equipo patrón: patrón para la comparación de los instrumentos.

- Costos por frecuencia de calibración: según el proceso y el instrumento a medir se tiene presente el número de calibraciones.

- Costos por transporte: flete del envío para calibrar aquellos equipos que necesiten calibrar por fuera de las instalaciones de la empresa.

\section{Costo de mantenimiento}

Solo aplican para los equipos electrónicos que se requiera intervenir por reparación o fallas, mantenimiento preventivo y garantías.

- Costos por fallas: existen muchos factores que implica la reparación de los instrumentos y según la magnitud se incrementa el costo.

\section{Costo de entrenamiento}

Los costos de entrenamiento es un gasto necesario teniendo en cuenta que el operador debe conocer al detalle el instrumento, además, hay que tener en cuenta que en los procesos hay alta rotación del personal y nuevas personas que ingresan al proceso.

Cuando las empresas no cuentan con este proceso de capacitación, existe alto margen de probabilidad que se genere mayores sobrecostos en la SAM.

\section{METOdOLOGÍA}

La metodología planteada para el desarrollo de la investigación se compone, en primera instancia, de una etapa exploratoria que tiene como objetivo el levantamiento de información puntual sobre el proceso productivo y sobre las variables de medición y control sobre las cuales se aplican los equipos en cuestión. En una segunda instancia, se desarrolla una etapa descriptiva que permite realizar un diagnóstico puntual para determinar el proceso más relevante, dependiendo de la cantidad de equipos de medición implicados en él y los requerimientos metrológicos del mismo. Finalmente, se llega a una etapa cuantitativa directa donde se obtiene información relevante para el sistema de aseguramiento metrológico de la compañía Renault-Sofasa, en un periodo de 5 años para su posterior análisis. A continuación, se describen particularmente cada una de las etapas de la metodología aplicada.

\section{Etapa 1}

En esta primera etapa el método de diagnóstico es exploratorio, donde se hace necesario conocer primero las actividades del proceso e identificar las variables de control, de este modo es posible realizar un análisis más claro y preciso. Para ello se realiza la 
caracterización de los tres procesos de producción donde interviene el sistema metrológico, los cuales son: el proceso de soldadura, pintura y ensamble.

\section{Etapa 2}

Posteriormente a la caracterización de cada proceso y con el suministro de los datos históricos de los últimos 5 años, se procede a determinar por medio del Principio de Pareto cuál es el proceso que más afecta el SAM, esto con el fin de poder intervenir específicamente el proceso seleccionado y proceder a realizar el diagnóstico.

\section{Etapa 3}

En esta etapa se utilizan herramientas de calidad aplicada en el mejoramiento continuo, el diagrama causa efecto o espina de pescado, permite identificar las causas principales que impactan negativamente al momento de dar de baja a un equipo, esta información será suministrada por parte de Sofasa, según los registros de la base de datos.

\section{Etapa 4}

Luego de detectar cuáles son las causas que generan estos sobrecostos se cuantificará el costo total del periodo caso de estudio y el impacto generado en los rechazos de los equipos de medición, con este método se presentará la desviación económica negativa, que a su vez genera pérdidas económicas que afecta la rentabilidad de la compañía. Los costos para analizar son: costos de adquisición, costos por entrenamiento, costos por operación, costos por calibración, costos por mantenimiento.

\section{Etapa 5}

Finalmente, se elabora un análisis del diagnóstico obtenido que permite realizar unas propuestas de posibles acciones de mejoramiento que impactarán directamente en el SAM de la compañía.

\section{RESULTADOS Y DISCUSIÓN}

Teniendo en cuenta los registros de los años comprendidos entre el periodo 2011-2015, se procede analizar cuántos equipos en total se dieron de baja, para esto se aplicó el diagrama de Pareto. A continuación, en la Fig. 1 se representa el total de los equipos dados de baja.

Según la Fig. 1 se puede evidenciar que aproximadamente cada año en promedio se presentan un $20 \%$ de equipos de medición dados de baja, incluso en los últimos dos años el incremento ha tenido mayor impacto.

En total se han utilizado 3862 equipos durante los últimos 5 años, de los cuales 753 equipos son dados de baja, es decir un $20 \%$ en promedio por año.

Después de determinar la cantidad total de los equipos que fueron retirados, se procede a identificar cuál es el proceso del departamento de producción que generó más impacto al momento de dar de baja los equipos.

Por medio de la siguiente figura se presenta la cantidad de casos por cada proceso y se evidencia el proceso que más afecta el SAM.

En la Fig. 2, se puede observar que el proceso de pintura representa el $50 \%$ de los equipos dados de baja, seguido por el proceso de ensamble con un $30 \%$ y por último el proceso de soldadura con un $20 \%$.

Según los resultados, se concluye que el proceso más crítico y el que presenta mayor impacto al momento de retirar un equipo de medición en el periodo comprendido entre los años 2011 a 2015 es el proceso de pintura, es importante aclarar que el proceso de pintura es uno de los procesos más complejos y controlados de Renault Sofasa, por sus altos estándares de calidad en sus vehículos. 
Se procede a conocer los factores que posiblemente inciden en la exclusión de los equipos en el proceso de pintura, según un diagrama de causa efecto: análisis de los factores que incide dar de baja un equipo de medición.
Se ejecuta el análisis correspondiente al proceso de pintura para determinar la causa raíz de los factores que inciden el momento de dar de baja un equipo.

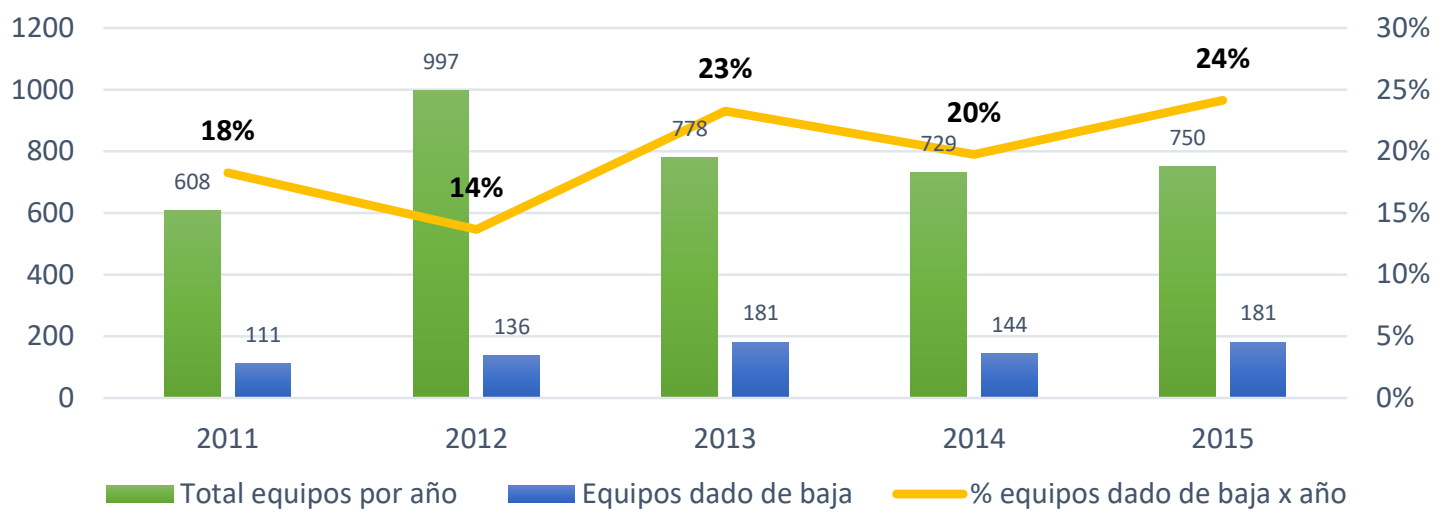

Figura 1. Total, equipos vs equipos dados de baja por año

Figure 1. Total, teams vs. teams given off per year Fuente: elaboración propia.

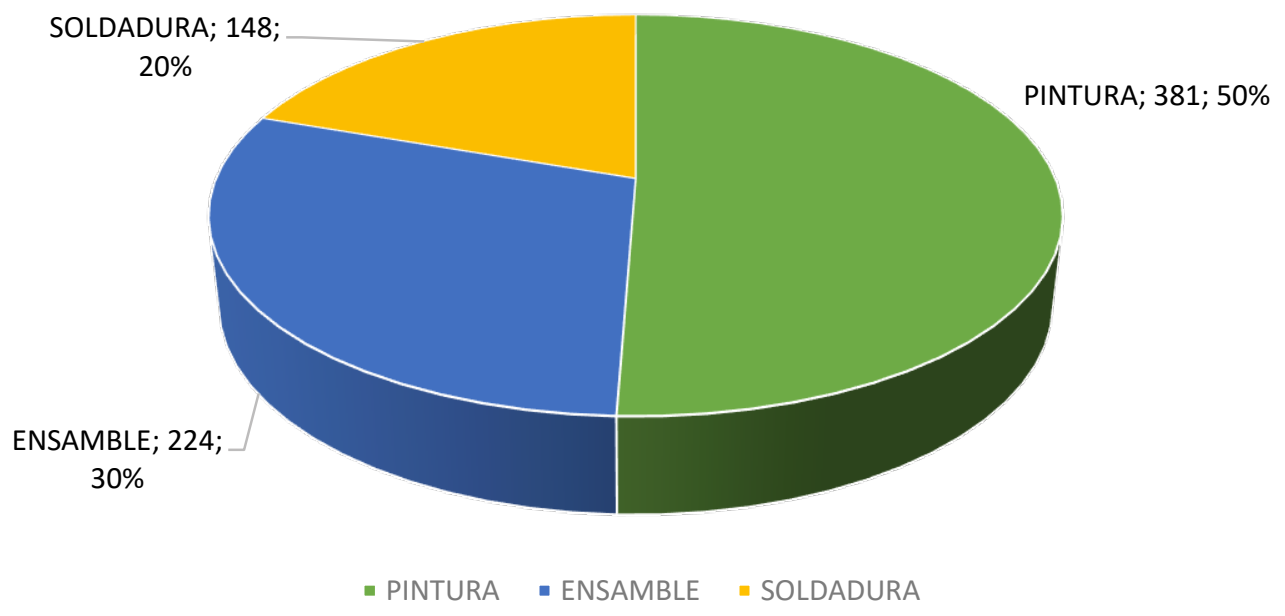

Figura 2. Total, equipos dado de baja por proceso en los últimos 5 años

Figure 2. Total equipment discharged per process in the last 5 years Fuente: elaboración propia. 


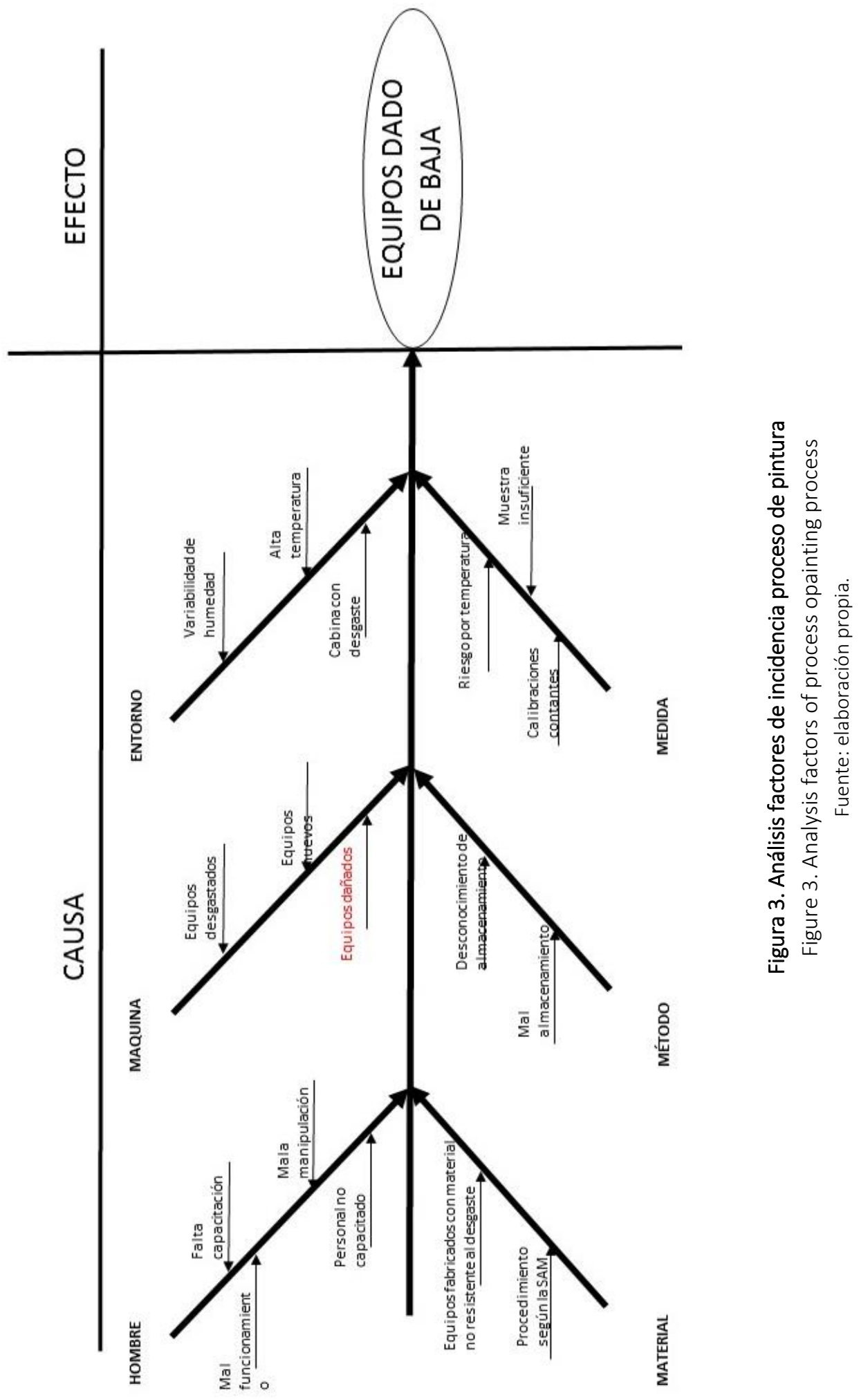


Después de analizar las causas tomando como referencia el diagrama de espina de pescado, se tienen los siguientes resultados.

La Tabla 1 corresponde al análisis de lectura de los factores que inciden al dar de baja los equipos de medición, solo en este proceso. Permite a los encargados de controlar el SAM de Renault Sofasa, determinar la causa raíz del problema y de esta forma buscar soluciones para mejorar y controlar el SAM del proceso de pintura.

Se presenta el análisis de incidencia en los equipos de pintura, en total son 381 instrumentos que se dieron de baja.

Tabla1. Lectura de factores de incidencia

Table 1. Reading of incidence factors

\begin{tabular}{ll}
\hline \multicolumn{1}{c}{ Lectura factores de incidencia } \\
\hline Daño del instrumento & $\begin{array}{l}\text { Instrumento dañado: por la mala manipulación por parte del operador, } \\
\text { por utilizar el instrumento en un proceso no adecuado. }\end{array}$ \\
\hline Retiro del proceso & $\begin{array}{l}\text { Instrumento se retira del proceso porque ya no se necesita para su } \\
\text { control, o por compra innecesaria. }\end{array}$ \\
\hline Pérdida & Instrumento perdido. \\
\hline Índice de calidad & $\begin{array}{l}\text { Instrumentos sin parámetros de control: equipos sin escala de medición, } \\
\text { equipos sin ficha técnica, etc. }\end{array}$ \\
\hline Mal funcionamiento & $\begin{array}{l}\text { Instrumento no claro en el proceso eje: desconocimiento del equipo vs } \\
\text { proceso }\end{array}$ \\
\hline Otros & Motivos no calificados o desconocidos por la fuente \\
\hline
\end{tabular}

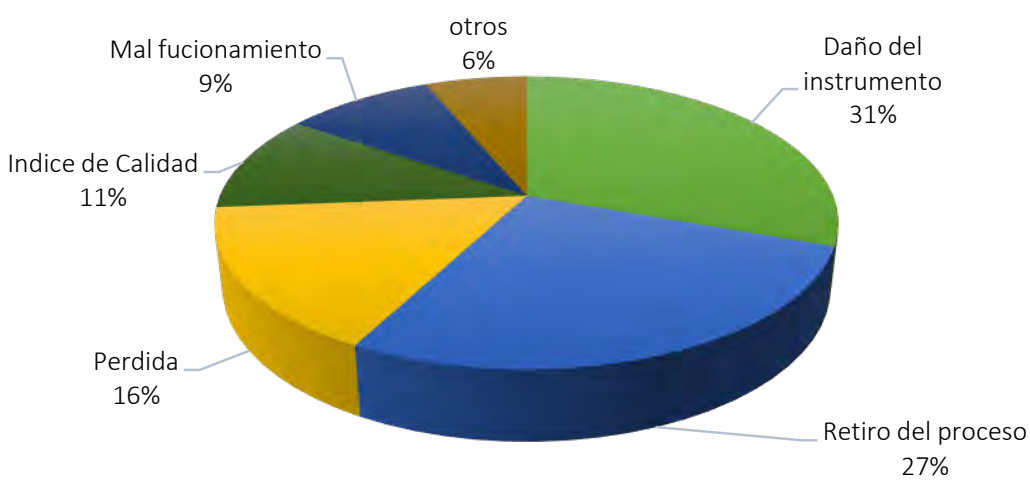

$\begin{array}{lll}\text { Daño del instrumento } & \text { Retiro del proceso } & \text { Perdida } \\ \text { Indice de Calidad } & \text { Mal fucionamiento } & \text { otros }\end{array}$

Figura 4. Factores de incidencia al momento de dar de baja los equipos de medición Figure 4. Incidence factors when the measuring equipment is removed Fuente: elaboración propia. 
Según los resultados, obtenidos en la Fig. 4, las dos causas más comunes al momento de dar de baja un equipo de medición en el proceso de pintura es por daño del instrumento y el retiro del equipo del proceso con una participación del $31 \%$ y $27 \%$ correspondiente; adicionalmente también hay otras causas, como la pérdida del equipo con un $16 \%$, por índice de calidad con un $11 \%$ de participación, por mal funcionamiento de los equipos con un $9 \%$ y por último con una participación del $6 \%$ otras razones.

Después de determinar el proceso de pintura como más crítico en el departamento de producción y conocer los factores de incidencia que afectan dar de baja los instrumentos de medición, se procede a identificar el costo total de cada equipo dado de baja, para el caso de pintura se cuenta con 381 equipos durante los últimos 5 años, comprendidos en los periodos 2011-2015.

Para calcular estos costos totales se tuvo en cuenta la recomendación del autor Jaime Restrepo «Costos del Aseguramiento Metrológico» (SAM).

La Fig. 4 representa los sobre costos totales del proceso de pintura en los últimos 5 años, con 242.336 millones de pesos (moneda local colombiana), se tienen en cuenta los 5 costos de SAM. Ver Tabla 3.

Adicionalmente, se procede a identificar el instrumento con mayor impacto económico que está generando mayor pérdida y se tiene el siguiente resultado según la Fig. 5.

Tabla 2. Lectura de costos totales

Table 2. Total cost reading

\section{Lectura análisis de costos totales}

\begin{tabular}{ll}
\hline Cantidad de equipos & Número de equipos dados de baja \\
\hline Costo de equipo & Costo de adquisición de todos los equipos dados de baja \\
\hline $\begin{array}{l}\text { Costo x mantenimiento } \\
\text { (MMT) }\end{array}$ & Costo total de mantenimiento x total equipo \\
\hline Costo de entrenamiento & $\begin{array}{l}\text { Costo generado por el entrenamiento al personal operativo, esta } \\
\text { capacitación solo aplica para algunos instrumentos }\end{array}$ \\
\hline Costo de calibración & $\begin{array}{l}\text { Costo de la calibración de los equipos, algunos son calibraciones } \\
\text { externas y otras son realizadas por la persona responsable del SAM }\end{array}$ \\
\hline Costo de operación & $\begin{array}{l}\text { Corresponde al recurso utilizado para utilizar cada instrumento } \\
\text { Cálculo del costo de } \\
\text { entrenamiento }\end{array}$ \\
\hline Cálculo del costo de operación de la persona que ejecuta la capacitación para el caso de Renault el \\
costo por hora es de \$ 100.000
\end{tabular}

Fuente: elaboración de los autores. 


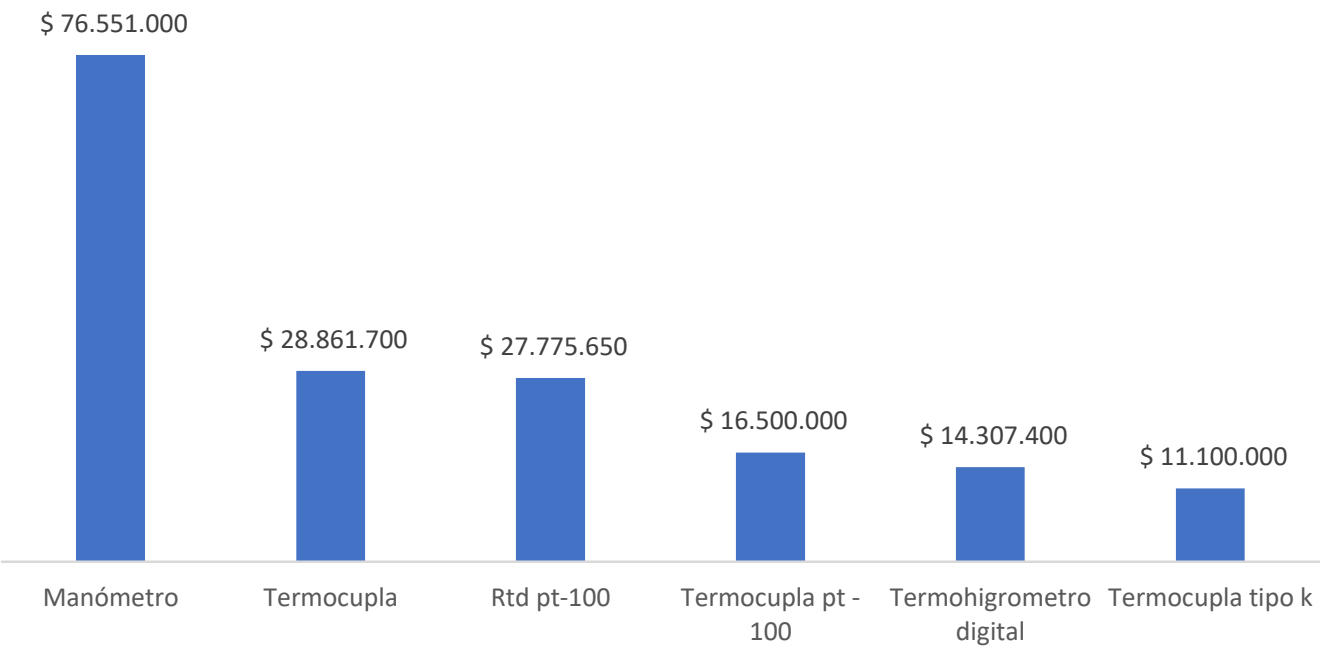

Figura 5. Top 5 de equipos con mayores sobrecostos al momento de dar de baja en el proceso de pintura Figure 5. Top 5 of equipment with higher overcharges at the time of being removed from the painting process Fuente: elaboración de los autores.

De acuerdo con la Fig 5 del costo total de los equipos dados de baja, en el proceso de pintura en los últimos 5 años, el instrumento que aparece fuera de control es el manómetro con un sobrecosto de 76,5 millones de pesos con un $30 \%$.

Para finalizar, se estima que el costo total de los sobrecostos de Renault es de 500.000 millones de pesos en los 3 procesos durante los últimos 5 años, claramente se evidencia la importancia de conocer estos sobrecostos, lo que le permite a la empresa tomar los correctivos necesarios e incrementar su rentabilidad al no incurrir nuevamente en estos sobrecostos.

En la etapa final partiendo de la metodología y los resultados obtenidos, se realizan las recomendaciones que pueden ser acogidas por la empresa para la intervención de las causales de los sobrecostos en los equipos de medición. Las recomendaciones planteadas son las siguientes:
- Capacitación del personal. Se recomienda implementar un plan de acción de capacitación del personal, debido a que se encontró un gran porcentaje de operarios de la compañía que no cuentan con las competencias pertinentes para manipular los instrumentos de medición, de este modo muchos de estos rechazos se presentaban por mala ejecución.

- Sectorización el uso de instrumentos de medición por departamentos. Al no existir un control de uso del instrumento sectorizado, los instrumentos se utilizan para realizar mediciones a múltiples etapas del proceso, exponiendo a los equipos a los diversos riesgos de cada etapa de la cadena de producción. Por lo anterior, se recomienda realizar la planeación sectorizada por departamento donde el instrumento es limitado para su funcionamiento. 
Tabla 3. Costo total de equipos dados de baja en el proceso de pintura Table 3. Total cost of equipment discharged in the painting process

\begin{tabular}{|c|c|c|c|c|c|c|c|}
\hline Equipo & $\begin{array}{l}\text { Cantidad } \\
\text { de } \\
\text { equipos }\end{array}$ & $\begin{array}{l}\text { Costo del } \\
\text { equipo }\end{array}$ & $\begin{array}{l}\text { Costo de } \\
\text { mantenimiento }\end{array}$ & $\begin{array}{c}\text { Costo } \\
\text { entrenamiento }\end{array}$ & $\begin{array}{l}\text { Costo de } \\
\text { calibración }\end{array}$ & $\begin{array}{l}\text { Costo de } \\
\text { operación }\end{array}$ & Total \\
\hline Rtd pt- 100 & 19 & 21.025 .650 & 1.900 .000 & & 3900.000 & 950.000 & 27.775 .650 \\
\hline Beaker & 1 & 11.200 & 100.000 & & 90.000 & 50.000 & 251.200 \\
\hline Bureta de $25 \mathrm{ml}$ & 10 & 782.000 & 1.000 .000 & & 900.000 & 500.000 & 3.182 .600 \\
\hline $\begin{array}{l}\text { Comparador de } \\
\text { carátula }\end{array}$ & 2 & 320.000 & 200.000 & 1.000 .000 & 320.000 & 100.000 & 1.940 .000 \\
\hline $\begin{array}{l}\text { Cono de } \\
\text { sedimentación }\end{array}$ & 2 & 351.600 & 200.000 & & 180.000 & 100.000 & 831.600 \\
\hline Copa ford $n^{\circ} 4$ & 3 & 1.809 .000 & 300.000 & & 459.000 & 150.000 & 2.718 .000 \\
\hline Copa ford & 3 & $1,292.000$ & 300.000 & & 459.000 & 150.000 & 2.201 .000 \\
\hline Cronómetro digital & 32 & 2.570 .000 & 3.200 .000 & 600.000 & 1.280 .000 & 1.600 .000 & 9.250 .000 \\
\hline Espectrofotómetro & 2 & 6.000 .000 & 200.000 & & 600.000 & 100.000 & 6.900 .000 \\
\hline Galga en $\vee$ para ajustes & 13 & 2.330 .000 & 1.300 .000 & & 949.000 & 650.000 & 5.229 .000 \\
\hline $\begin{array}{l}\text { Juego de galgas en } \\
\text { bloque }\end{array}$ & 1 & 300.000 & 100.000 & & 73.000 & 50.000 & 523.000 \\
\hline Manómetro & 158 & 27.826 .000 & 15.800 .000 & 1.000 .000 & 24.025 .000 & 7.900 .000 & 76.551 .000 \\
\hline $\begin{array}{l}\text { Medidor de brillo } \\
\text { ángulo } 20^{\circ}\end{array}$ & 1 & 200.000 & 100.000 & & 117.000 & 50.000 & 467.000 \\
\hline Medidor de espesores & 3 & 6.000 .000 & 300.000 & 400.000 & 351.000 & 150.000 & 7.201 .000 \\
\hline Medidor de nivelación & 1 & 1.000 .000 & 100.000 & & & 50.000 & 1.150 .000 \\
\hline Pipeta graduada $10 \mathrm{ml}$ & 1 & 25.000 & 100.000 & 200.000 & 90.000 & 50.000 & 465.000 \\
\hline $\begin{array}{l}\text { Pipeta volumétrica de } \\
10 \mathrm{ml}\end{array}$ & 3 & 79.000 & 300.000 & & 270.000 & 150.000 & 799.000 \\
\hline $\begin{array}{l}\text { Pipeta volumétrica de } \\
1 \mathrm{ml}\end{array}$ & 5 & 124.000 & 500.000 & & 450.000 & 250.000 & 1.324 .000 \\
\hline $\begin{array}{l}\text { Pipeta volumétrica de } \\
5 \mathrm{ml}\end{array}$ & 1 & 26.000 & 100.000 & & 90.000 & 50.000 & 266.000 \\
\hline $\begin{array}{l}\text { Probeta plástica de } \\
250 \mathrm{ml}\end{array}$ & 33 & 594.000 & 3.300 .000 & & 2.970 .000 & 1.650 .000 & 8.514 .000 \\
\hline Regla graduada $500 \mathrm{ml}$ & 1 & 16.500 & 100.000 & & 73.000 & 50.000 & 239.500 \\
\hline Rugosímetro & 3 & 200.000 & 100.000 & & & 150.000 & 450.000 \\
\hline Termocupla & 18 & 19.961 .700 & 1.800 .000 & 8.00 .000 & 5.400 .000 & 900.000 & 28.861 .700 \\
\hline Termocupla pt 100 & 10 & 12.000 .000 & 1.000 .000 & & 3.000 .000 & 500.000 & 16.500 .000 \\
\hline Termocupla tipo j & 2 & 2.400 .000 & 200.000 & & 600.000 & 100.000 & 3.300 .000 \\
\hline Termocupla tipo k & 6 & 7.200 .000 & 600.000 & 1.200 .000 & 1.800 .000 & 300.000 & 11.100 .000 \\
\hline Termógrafo & 1 & 1.200 .000 & 100.000 & & 300.000 & 50.000 & 1.650 .000 \\
\hline $\begin{array}{l}\text { Termohigrómetro } \\
\text { digital }\end{array}$ & 23 & $6.224,400$ & 2.300 .000 & 700.000 & 3.933 .000 & 1.150 .000 & 14.307 .400 \\
\hline $\begin{array}{l}\text { Termómetro de } \\
\text { caratula }\end{array}$ & 8 & 1.095 .000 & 800.000 & & 584.000 & 400.000 & 2.879 .000 \\
\hline Termómetro de vidrio & 14 & 505.000 & 1.400 .000 & & $1,022.000$ & 700.000 & 3.627 .000 \\
\hline Torquímetro & 1 & 1.000 .000 & 100.000 & 600.000 & 133.000 & 50.000 & 1.883 .000 \\
\hline Piezas total & 381 & & & & & & 242.336 .050 \\
\hline
\end{tabular}

Nota. Los valores de los costos están dados en miles de pesos. Fuente: elaboración de los autores. 
- Restructuración del almacenamiento del instrumento de medición. Se propone la creación por cada proceso de una zona de almacenamiento para todos los equipos de medición, garantizando condiciones óptimas y almacenamiento y conservación de los equipos.

- Control de adquisición de equipos. Debido a que cada proceso tiene la libertad de adquirir cualquier equipo de medición para controlar sus procesos, no se garantiza que los instrumentos adquiridos por la empresa sean los apropiados para cada aplicación. Se recomienda limitar las compras de cualquier equipo de medición para que solo sean adquiridos con el visto bueno de la persona responsable de metrología, así cada equipo de medición adquirido será utilizado para su uso previsto.

- Asignación de personas de apoyo para controlar los procesos. Al momento la compañía cuenta con solo una persona responsable para realizar el control metrológico en los tres procesos de producción. Se recomienda asignar personal calificado adicional que permita la administración del SAM de una manera más eficiente.

\section{CONCLUSIONES}

Esta investigación permitió a la compañía Renault Sofasa conocer el estado real del sistema de aseguramiento metrológico y los costos inherentes al sistema de medición; así mismo, los registros obtenidos del periodo 2011-2015, permitieron a los investigadores elaborar el diagnóstico de los sobrecostos a través de herramientas de calidad. Se determinó que de los 3 procesos de producción más relevantes en la empresa el que aporta mayor número de equipos retirados es el proceso de pintura. Dicho proceso aporta el 50 $\%$ de equipos dados de baja, lo que le significó a la compañía un sobrecosto de 242.336 millones de pesos en el periodo anteriormente mencionado.

Por medio de la investigación se determinó que el factor que tiene mayor incidencia para dar de baja los equipos se encuentra asociado a daños irreparables causados al mismo. En promedio el $31 \%$ de los equipos son dados de baja por este factor, adicionalmente las magnitudes más relevantes al momento de dar de baja los equipos de medición en el proceso de pintura son presión y temperatura.

Se presentó a los líderes de la empresa, un documento con las recomendaciones propuestas que se espera sean tenidas en cuenta por la alta dirección como herramienta para la toma de decisiones a futuro que permitan la disminución del retiro de los equipos de medición asociados a las magnitudes anteriormente identificadas en el proceso de pintura. Adicionalmente, se recomienda a la compañía aplicar la metodología empleada en esta investigación a los demás procesos con el fin de conocer los sobrecostos a los que el sistema productivo se enfrenta y con esto implementar acciones que les permita mejorar su sistema de medición y minimizar sus costos. Así mismo, se recomienda replicar un estudio similar en las empresas que utilicen instrumentos de medición en etapas críticas de sus procesos, lo cual posibilitará conocer los sobrecostos a los que pueden estar expuestos en su sistema de aseguramiento metrológico, permitiéndoles corregir y mejorar su rentabilidad en sus procesos productivos. 


\section{REFERENCIAS}

ANDI \& FENALCO. (2015). Informe del sector automotor.

Chirinos, E., Rivero, E., \& Mendez, E. (2015). El kaizen como un sistema actual de gestión personal para el éxito organizacional en la empresa ensambladora Toyota. Revista Científica y Electrónica Ciencias Gerenciales, 16(5), 113-135.

FENALCO. (2017). Informe del sector automotor a diciembre de 2017.

Hernández, T., Oropesa, P., \& Serra, R. (2012). Aseguramiento metrólogico en la producción y uso de radiofármacos. Nucleus, 52, 20-24.

ICONTEC. (2002). NTC-ISO 17025: Requisitos generales de competencia de laboratorios de ensayo y calibración. Icontec.

ICONTEC. (2003). Norma técnica colombiana NTC-ISO 10012: sistemas de gestión de la medición requisitos para los procesos de medición y los equipos de medición. Icontec.

Imai, M. (2003). Cómo implementar el Kaizen en el sitio de trabajo. Santafé de Bogotá: McGraw-Hill.

ISO. (2007). OHSAS 18001:2007. En sistemas de gestión en seguridad y salud.

ISO. (2008). Norma internacional ISO 9001.
ISO. (2015). Norma ISO 14001. En sistemas de gestión ambiental.

Jimenez, L. (2007). Metrología industrial, sistemas de medición y aseguramiento metrológico: conceptos fundamentales.

Kume, H. (1992). Herramientas estadísticas básicas para el mejoramiento de la calidad. Editorial Norma.

Lopez, A., Amezola, R., \& Elías, A. (2007). Aseguramiento metrológico en los ensayos no-destructivos por ultrasonido. In 7a Confrencia Mexicana de Pruebas No Destructivas. Cd. Mexico.

Maarof, M., \& Mahmud, F. (2016). Review of contributing factors and challenges in implementing Kaizen in small and medium enterprises. Procedia Economics and Finance, 522-531.

Para Conesa, J. E. (2007). Kaize: cuando la mejora se hace realidad. Técnica Industrial, 271, 31-35.

Restrepo, J. (2016). Metrología aplicada. Medellín.

Rodríguez, E. (2013). Aseguramiento metrológico para Equipos médicos. IFMBE Proceedings, 33, 491-494.

Unger, K. (2004). La industria automotriz en tres reigones de México.Un análisis de clústers. El Trimestre Económico, 909-941. 